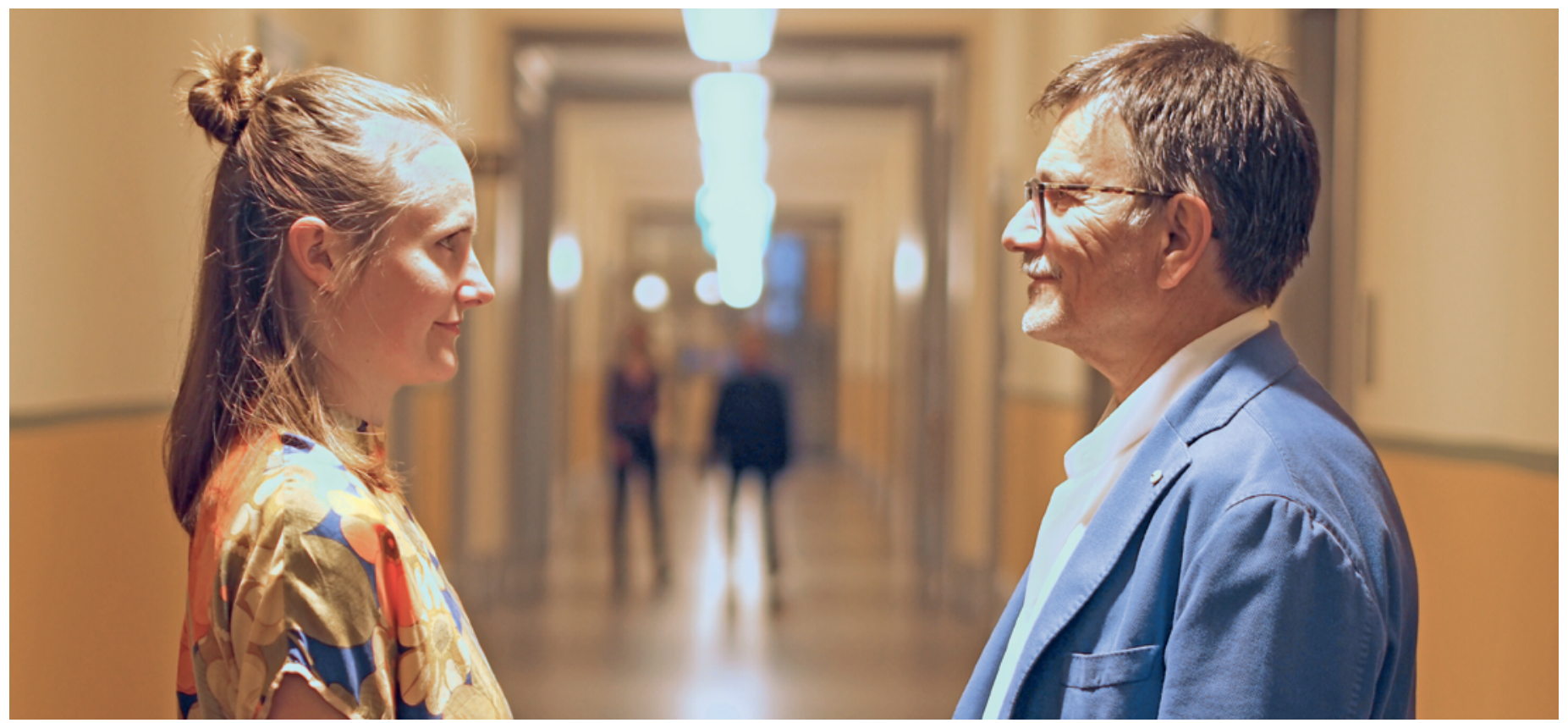

Willst du? Soll ich? Lisa Bircher und Bruno Kissling reflektieren in ihrem gemeinsamen Buch über Medizin und Arztsein.

\title{
«Medizin ist mehr als das Machbare»
}

\section{Daniel Lüthi}

Freier Journalist und Fotograf, Medientrainer, Bern

\section{Lisa Bircher und Bruno Kissling haben in Bern ihr gemeinsames neues Werk vorgestellt: das Buch "Ich stelle mir eine Medizin vor ..."}

Die Einleitung dieses Büchleins fasst seinen Plot so zusammen: «Lisa, eine junge Ärztin am Anfang ihres ärztlichen Wirkens, und Bruno, ein erfahrener Hausarzt am Ende seiner Berufszeit, schreiben sich während des Zeitraums von eineinhalb Jahren Briefe.» Inhaltliche Zusammenfassung an der Vernissage: «Der Mensch muss wieder mehr ins Zentrum der Medizin kommen.»

\section{Selbstverständlichkeiten?}

Eigentlich sind es Banalitäten: Medizin sollte nicht Selbstzweck sein, es geht um den Patienten. Und: Arzt und Ärztin sind auch Menschen. Deshalb sollten sie sich selber ebenso ernst nehmen wie ihre Patientinnen und Patienten.

In diesem Buch aber legen eine Ärztin am Anfang ihrer Karriere und ein Arzt an deren Ende in einem langen

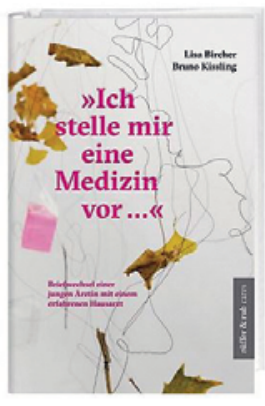

Lisa Bircher, Bruno Kissling "Ich stelle mir eine Medizin vor ..." Briefwechsel einer jungen Ärztin mit einem erfahrenen Hausarzt

144 Seiten.

Zürich: Rüffer \& Rub; 2018. ISBN 978-3-906304-39-7

Dialog Zeugnis davon ab, wie schwierig und oft auch schmerzhaft es in diesem Beruf ist, solche Banalitäten im Alltag zu leben. Und wie wenig selbstverständlich es ist, dass sich Mediziner in ihrem hektischen Alltag über Grundsätzliches Gedanken machen und sich darüber austauschen. Der im Buch dokumentierte Dialog über die Generationengrenze hinweg ist unter anderem diesem Manko zu verdanken.

Schon in ihrem ersten Brief an Bruno wundert sich Lisa sehr: «Zu Beginn war ich dermassen mitgenom- 


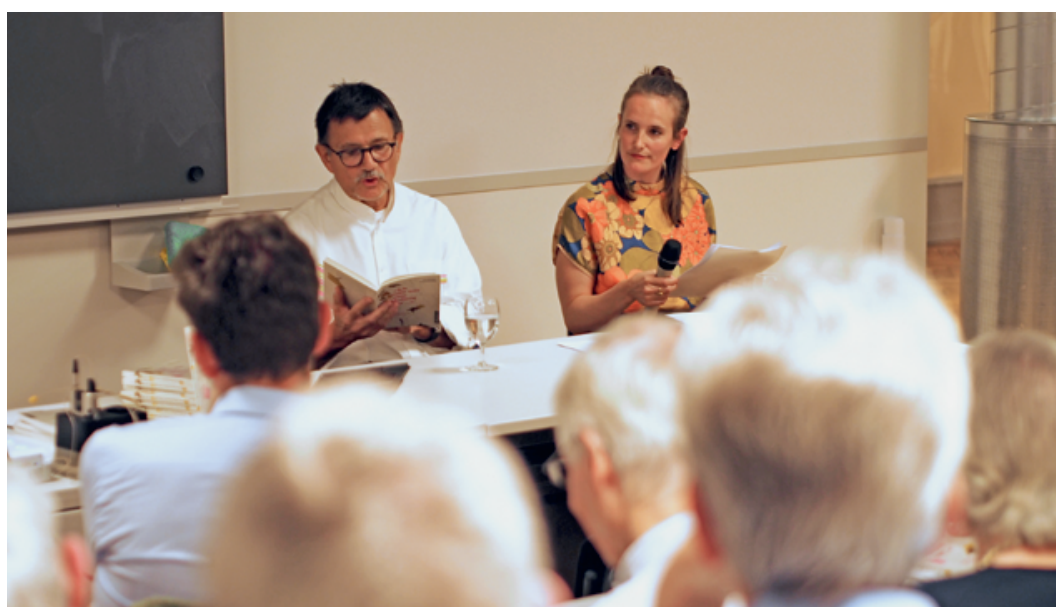

Für die Buchvernissage wählten die Autoren das Institut für Hausarztmedizin der Universität Bern.
Wandel, sagt sie im persönlichen Gespräch an der Vernissage im Institut für Hausarztmedizin in Bern. Kinder- und Jugendpsychiatrie wäre allenfalls eine Option. Vorerst weilt sie im Mutterschaftsurlaub, dann sehe sie weiter.

Und Bruno? Er steht vor dem Ende seines Berufslebens. Zurzeit ist er noch zu 50\% in seiner ehemaligen Praxis angestellt. «Wenn ich einen Nachfolger habe, höre ich auf», sagt er. Da schwingt Erleichterung mit. Auch darüber, dass er die strengen Jahre ohne grösseren gesundheitlichen Schaden überstanden hat. Lisa wird nicht seine Nachfolgerin werden, wie gesagt. «Eigentlich schade», kommentiert Bruno.

Wenn die beiden Mediziner über ihren medizinischen Alltag sprechen, entsteht an dieser Vernissage also immer wieder eine bedenkenswert nachdenkliche Stimmung. Der Briefwechsel im gemeinsamen Büchlein erhellt die Gründe.

men vom Alltag in der Klinik, dass ich kaum je eine Sekunde übrig hatte, über den Tellerrand hinauszuschauen.» Bruno findet es gut, dass sie schon früh merkt, wo diese fachärztliche Weiterbildung krankt. Sie sei zu sehr "disease-bezogen", schreibt er. Kurz darauf berichtet Lisa, wie sie am Universitätsspital Lausanne selber fast krank geworden ist und wie sie ihren Job als Assistenzärztin fluchtartig verlassen hat: «Wir sind zu weit entfernt von den Patienten, zu sehr beschäftigt mit Untersuchungen und Behandlungen.»

\section{Differenzen?}

Keine Zeit, auch für sich selber nicht, kaum Kommunikation, dafür viel technischer Aktivismus: Die Themen bleiben. Und die beiden reflektieren Alternativen, suchen nach Inseln. Sie tun dies in einer oft rührenden Einigkeit. Gibt es auch Differenzen? Gab es Momente und Themen, wo die Meinungen und Empfindungen der beiden auseinandergingen? Im Gespräch schauen sie sich an, bevor sie antworten. Willst du? Soll ich? Uneinig seien sie sich vielleicht bei der Frage, ob im Kleinen oder im Grossen mehr zu erreichen sei, ist die konsolidierte Replik. «Mit dieser Publikation beispielsweise kann ich mehr Leute erreichen als in der ärztlichen Praxis - mehr Menschen, die Medizin anbieten, und mehr, die Medizin in Anspruch nehmen", sagt Lisa. Bruno war es als Hausarzt stets ein Anliegen, personenzentriert zu arbeiten. Als Standespolitiker hat er aber immer wieder auch versucht, Entwicklungen zu beeinflussen. «Bottom-up kann ich mehr erreichen als top-down», ist er heute überzeugt.

\section{Und jetzt?}

Lisa will nicht mehr Hausärztin werden. Sie wolle sich von der körperbezogenen Medizin abwenden, zunehmend interessiere sie sich für den gesellschaftlichen

\section{Bildnachweis}

Fotos: Daniel Lüthi

\section{Lisa Bircher}

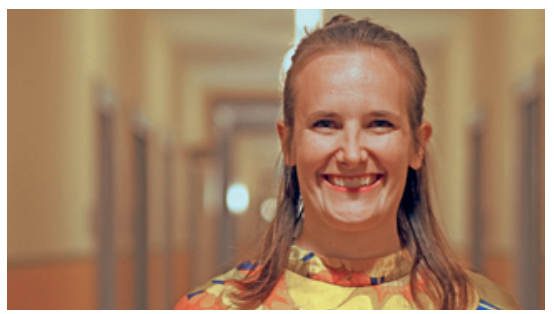

Lisa Bircher wurde 1986 in Bern geboren, wo sie auch Medizin studierte. 2012 schloss sie ihr Studium ab. Ihre berufliche Laufbahn startete in der Inneren Medizin mit dem Ziel, Hausärztin zu werden. Während zweier Jahre engagierte sie sich beim Verband Schweizerischer Assistenz- und Oberärztinnen und -ärzte (VSAO). Zurzeit weilt sie im Mutterschaftsurlaub. Sie lebt in Basel.

\section{Bruno Kissling}

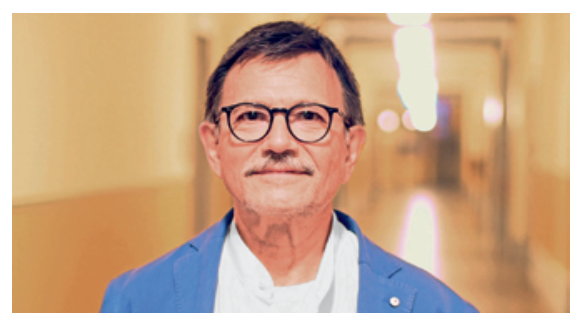

Bruno Kissling wurde 1949 geboren, seit 1982 ist er Hausarzt in Bern. Er war Vorstandsmitglied der Schweizerischen Gesellschaft für Allgemeinmedizin SGAM (1995-2003), Schweizer Delegierter beim Welthausärzteverband WONCA (2000-2009), Mitbegründer und Chefredaktor bzw. Ko-Chefredaktor von «Primary and Hospital Care» (2001-2014) sowie Lehrarzt des Berner Instituts für Hausarztmedizin. Mit der Medizinanthropologin Andrea Abraham veröffentlichte er 2015 das Buch "Qualität in der Medizin Briefe zwischen einem Hausarzt und einer Ethnologin". 